Copyright by the Acoustical Society of America. Hinich, M. J. (1979). On errors in some papers on array processing. Journal of the Acoustical Society of America, 65(2), 530-531. doi: 10.1121/1.382310

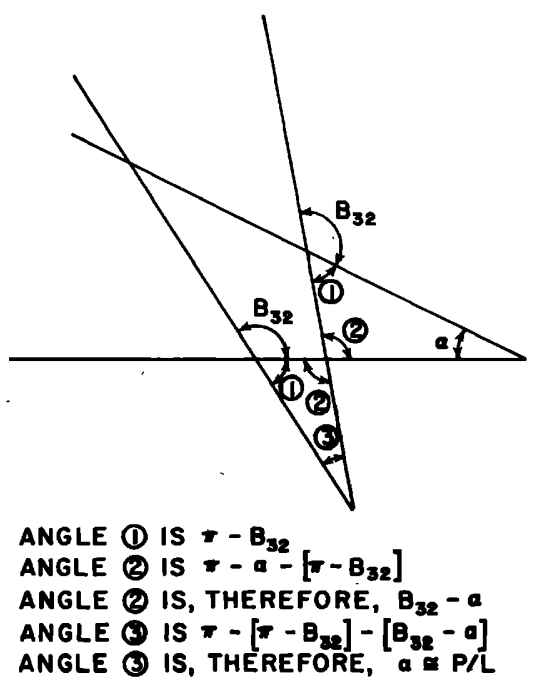

FIG. 4. Difference in estimated and true bearing angles given approximately by $P / L$.

when the bearing errors are uncorrelated. However, if the bearing errors are highly correlated, as in the case of the midarray deflecting from noncollinearity, Eq. (6) must be modified and the relative range error is given approximately by

$$
\begin{aligned}
& \frac{\sigma(\hat{R})}{R} \simeq \frac{2 R}{L_{e}} \cdot \frac{\sigma(\hat{P})}{L}, \\
& \simeq \frac{2 R}{L_{e}} \sigma(\hat{B}),
\end{aligned}
$$

where $L_{e}$ is the effective interarray separation, $L \sin B_{2}$ ( $L$ is half of the total array length), and $\sigma(\hat{P})$ is the standard deviation of the midarray deflection from a line connecting the forward and after arrays (either in absolute terms or in the amount that cannot be calibrated out).
Equation (7) is consistent with results of Carter, ${ }^{8}$ Hahn, ${ }^{9}$ and Beam. ${ }^{10}$ Therefore, we conclude that the approximation, Eq. (7), is reasonable.

\section{CONCLUSIONS}

The similarities of wavefront-curvature and triangulation techniques as methods for passively estimating the range to an acoustic source have been demonstrated. These similarities have been used to extend previous triangulation results to predict the range errors because of uncertainty in the position of array hydrophones. The extended triangulation results agree with previous optimum theory.

\section{ACKNOWLEDGMENTS}

The author acknowledges several helpful discussions with Drs. N. Yen, J. Beam, D. Bordelon, W. Hahn, and R. M. Kennedy.

${ }^{1}$ H. E. Fridge (private communication, 1973).

${ }^{2}$ G. C. Carter (working papers, 1976).

${ }^{3}$ J. B. Hall, Jr., and R. I. Hayford, Jr. (private communication, 1977).

${ }^{4}$ W. J. Bangs and P. M. Schultheiss, "Space-time Processing for Optimal Parameter Estimation," in Signal Processing, edited by J. W. R. Griffiths, P. L. Stocklin, and C. van Schoonveld (Academic, New York, 1973), pp. 577-590.

${ }^{5}$ M. J. Hinich, "Bearing Estimation Using a Perturbed Linear Array," J. Acoust. Soc. Am. 61, 1540-1544 (1977).

${ }^{6} \mathrm{H}$. P. Bucker, "Beamforming a Towed Line Array of Unknown Shape," J. Acoust. Soc. Am. 63, 1451-1454 (1978).

${ }^{7}$ R. M. Kennedy (private communication, 1977).

${ }^{8}$ G. C. Carter, "Variance Bounds for Passively Locating an Acoustic Source with a Symmetric Line Array," J. Acoust. Soc. Am. 62, 922-926 (1977).

${ }^{9}$ W. R. Hahn (private communication, 1973) [however, see also W. R. Hahn, "Optimum Signal Processing for Passive Sonar Range and Bearing Estimation," J. Acoust. Soc. Am. 58, 201-207 (1975)].

${ }^{10} \mathrm{~J}$. Beam (private communication, 1978).

\title{
On errors in some papers on array processing
}

\author{
Melvin J. Hinich \\ Virginia Polytechnic Institute and State University, Blacksburg, Virginia 24061 \\ (Received 14 September 1978)
}

Some errors in the literature are noted concerning the calculation of the maximum-likelihood estimator.

PACS numbers: 43.60.Gk

Expressions (7) and (8) in my "Processing Spatially Aliased Arrays"1 paper are not correct. The maximumlikelihood estimator of bearing is

$$
\tilde{\theta}=w \sum_{l=1}^{B} \rho\left(\omega_{l}\right) \lambda^{-2}\left(\omega_{l}\right) \hat{\theta}\left(\omega_{l}\right),
$$

where $w=\left[\sum_{l=1}^{B} \rho\left(\omega_{l}\right) \lambda^{-2}\left(\omega_{l}\right)\right]^{-1}$. The asymptotic mean square error of $\tilde{\theta}$ is

$$
\mathrm{mse} \tilde{\theta}=\frac{3 w}{2 M L^{2}(\pi \sin \theta)^{2}} .
$$

The sub-bandwidths $\omega_{l+1}-\omega_{l}=\Delta \omega$ must be greater than $2 \pi / N \delta$. Let $\Delta \omega=2 \pi / m(N) \delta$ where $m \rightarrow \infty$ and $m / N \rightarrow 0$ as $N \rightarrow \infty$. For large $N$,

$$
w^{-1}=\frac{N}{2 \pi m} \int_{\omega_{a}}^{\omega_{b}}|A(\omega)|^{2} S_{\epsilon}^{-1}(\omega) c^{-2}(\omega) \omega^{2} d \omega,
$$

where $c(\omega)$ is the phase velocity and $|A|^{2} S_{6}^{-1}$ is the basic SNR. The broadband maximum-likelihood estimators of bearing and velocity in the article by Clay, Shaman, and Hinich are also incorrect. ${ }^{2}$ We normalized by the standard deviation instead of the variance. For constant 
$c$ and $\rho$, the ratio of the mse of our estimators to the mse of the maximum-likelihood estimators is $\left(\int_{\omega_{a}}^{\omega_{b}} \omega d \omega\right)^{2}\left[\left(\omega_{b}-\omega_{a}\right) \int_{\omega_{a}}^{\omega_{b}} \omega^{2} d \omega\right]^{-1}$. If $\omega_{a}=0$, this ratio is $3: 4$.

Note that mse $\ddot{\theta}$ is proportional to $m / N$ and not $1 / N$ as is stated by MacDonald and Schultheiss, ${ }^{3} \mathrm{Hahn},{ }^{4}$ and Carter. ${ }^{5}$ This agrees with the asymptotic variance derived by Hannan and Thompson. ${ }^{6}$

${ }^{1}$ M. J. Hinich, "Processing Spatially Aliased Arrays," J. Acoust. Soc. Am. 64, 792-794 (1978).
${ }^{2}$ C. S. Clay, M. J. Hinich, and P. Shaman, "Error Analysis of Velocity and Direction Measurements of Plane Waves Using Thick Large-Aperture Arrays," J. Acoust. Soc. Am. 53, 1161-1166 (1973).

${ }^{3}$ V. H. MacDonald and P. M. Schultheiss, "Optimum Passive Bearing Estimation," J. Acoust. Soc. Am. 46, 37-43 (1969).

${ }^{4}$ W. R. Hahn, "Optimum Signal Processing for Passive Sonar Range and Bearing Estimation," J. Acoust. Soc. Am. 58, 201-207 (1975).

${ }^{5}$ G. C. Carter, "Variance Bounds for Passively Locating an Acoustic Source with a Symmetric Line Array," J. Acoust. Soc. Am. 62, 922-926 (1977).

${ }^{6}$ E. J. Hannan and P. J. Thompson, "Estimating Group Delay," Biometrika 60, 241-253 (1973).

\title{
Comments on "Conformal array beam patterns and directivity indices" [J. Acoust. Soc. Am. 63, 841-847 (1978)
}

\author{
J. G. Kelly, E. J. Sullivan, and J. I. Salisbury \\ Weapons Systems Department, Naval Underwater Systems Center, Newport, Rhode Island 02840 \\ (Received 7 August 1978; revised 6 October 1978)

\begin{abstract}
their calculation of the Directivity Index (DI) of a particular conformal array. In addition, independent results of calculations of the DI for a similar conformal array (elements distributed on a conical surface) are presented. These results include optimal (maximal DI) weighting of the array's elements, as well as comparison with an appropriate planar array serving as a reference.
\end{abstract} \\ Several comments are presented in response to an article by Frank, Kesner, and Gruen which concern
}

PACS numbers: $43.60 . \mathrm{Cg}$

\section{INTRODUCTION}

In a recently published article, ${ }^{1}$ T. J. Frank, J.W. Kesner, and H. M. Gruen have advanced several misleading points which require comment. There are three in particular which we choose to address in this letter. These pertain to (1) their claim that their Eqs. (2)-(4) constitute alternative formulations of the directivity indexes (DI) of an array; (2) their claim that the DI of a uniformly weighted linear array is independent of the "scan angle" (that is, the steering angle); and (3) their choice of certain planar arrays as bases for comparison with their conformal array.

\section{DEFINITION OF DIRECTIVITY INDEX}

Regarding the first point, it is simply not so that their Eqs. (2)-(4) are alternative formulations of the expression for the DI. First of all, the DI defined by their Eq. (2) applies to both transmitting and receiving arrays; whereas the DI as defined by their Eqs. (3) and (4) can be meaningfully interpreted only for the case of receiving array. In either case, however, the expression given by their Eqs. (3) and (4) can be derived directly from that given by their Eq. (2). This mathematical equivalence has been recurrently demonstrated in the literature on acoustic and radar antenna design. ${ }^{2-4}$ Omitting algebraic details, we reiterate the derivation for the more general case of arbitrarily weighted point elements forming a volumetric (receiving) array.

The quantity $p(\theta, \phi)$, the plane wave array factor of an $N$ element receiving array, is given by

$$
p(\theta, \phi)=(1 / N) \sum_{n=1}^{N} h_{n} \exp \left[-i \mathbf{r}_{n} \cdot\left(\mathbf{k}-\mathbf{k}_{0}\right)\right],
$$

where $h_{n}$ is the (possibly complex) weighting or shading coefficient applied to the $n$th element's output, $\mathbf{r}_{n}$ is the position vector of the $n$th element output, $\mathrm{k}$ is the wave vector in the arbitrary direction $(\theta, \phi)$, and $\mathbf{k}_{0}$ is the wave vector in the steering direction $\left(\theta_{0}, \phi_{0}\right)$. The magnitude of both $\mathrm{k}$ and $\mathrm{k}_{0}$ is $k=2 \pi / \lambda$ where $\lambda$ denotes the wavelength; the spherical coordinates $(\theta, \phi)$ are defined in Fig. 1(a) following the convention used in Ref. 1.

The receive directivity function is defined by:

$$
b(\theta, \phi)=p(\theta, \phi) p^{*}(\theta, \phi),
$$

where * denotes complex conjugate. Therefore,

$$
b(\theta, \phi)=\left(1 / N^{2}\right) \sum_{m=1}^{N} \sum_{n=1}^{N} h_{m}^{*} h_{n} \exp \left[i\left(\mathbf{r}_{m}-\mathbf{r}_{n}\right) \cdot\left(\mathbf{k}-\mathbf{k}_{0}\right)\right] .
$$

The DI in the direction $\left(\theta_{0}, \phi_{0}\right)$ is defined ${ }^{5}$ by

$\mathrm{DI}=10 \log \left[4 \pi b\left(\theta_{0}, \phi_{0}\right) / \int_{0}^{2 \pi} \int_{-\tau / 2}^{\pi / 2} b(\theta, \phi) \cos \theta d \theta d \phi\right]$,

which is, of course, the array gain specialized to the case of a plane wave signal and isotropic noise in the farfield of the array. Substituting the above expression for $b(\theta, \phi)$ into Eq. (4), the DI is given by

$$
\begin{aligned}
\mathrm{DI}= & 10 \log \left\{\left|\sum_{n=1}^{N} h_{n}\right|^{2} /\right. \\
& \left.\sum_{m=1}^{N} \sum_{n=1}^{N} h_{m}^{*} h_{n} c_{m n} \exp \left[-i \mathrm{k}_{0} \cdot\left(\mathrm{r}_{m}-\mathbf{r}_{n}\right)\right]\right\},
\end{aligned}
$$

